\title{
Important Challenges in the Search for a Correct Characterization of Arbovirus Cases
}

\author{
Claudio Cesar Cirne Santos*, Caroline de Souza Barros and Izabel Christina Nunes de Palmer Paixão \\ Department of Cellular and Molecular Biology, Fluminense Federal University, Brazil
}

*Corresponding author: Claudio Cesar Cirne Santos, Department of Cellular and

Molecular Biology, Fluminense Federal University, Brazil.

Received Date: April 10, 2019

Published Date: April 22, 2019

\section{Commentary}

Arthropod-borne viruses (known as arboviruses) are widely circulated throughout the world, causing numerous episodes of emergence and resurgence of epidemic outbreaks with serious consequences to public health [1,2]. The arboviruses most frequently responsible for causing important outbreaks are distributed in the genera Alphavirus (family Togaviridae) including Chikungunya, Semliki Forest, Ross River, Mayaro virus and Sindbis virus [3]. Flavivirus (Flaviviridae family) such as Dengue, West Nile and Japanese encephalitis viruses are important to public health as well. Other viruses of consequence to human health are also reported, although less frequently. These include the Bunyaviridae, Reoviridae, and Rhabdoviridae.

Most arboviruses that cause human diseases have RNA genomes and are zoonoses that depend on wildlife or domestic animals for maintenance in nature; however, humans may be targets of these viruses. The identification of infections caused by such viruses can be determined by molecular techniques such as polymerase chain reaction (PCR), mainly in cases of acute febrile diseases [4], although many groups are having difficulty implementing techniques that are capable of differentiating infections [5] and considering possible co-infections between ZIKV and DENV and CHIKV $[6,7]$.

Although molecular detection is possible, the availability of these methodologies to the population at large during times of outbreak makes it impossible to determine precisely which virus is affecting an individual. Clinical manifestations are highly variable and may present from mild to more complex conditions with neurological complications, hemorrhage, and severe joint changes; causing a significant increase in the morbidity and mortality of these conditions [8]. The association between ZIKV infection with microcephaly and Guillain-Barré syndrome in 2015 brought fear and insecurity primarily to pregnant women, although it has caused concern among all people. In the same way, both DENV and CHIKV infection showed signs of worsening, although many people had been treated empirically because they manifested symptoms that are similar among different arboviruses; especially symptoms associated with dengue virus, Mayaro, Zika and Chikungunya such as fever, rash, muscular pains, joint pains, and headache, among others, and may only vary in intensity.

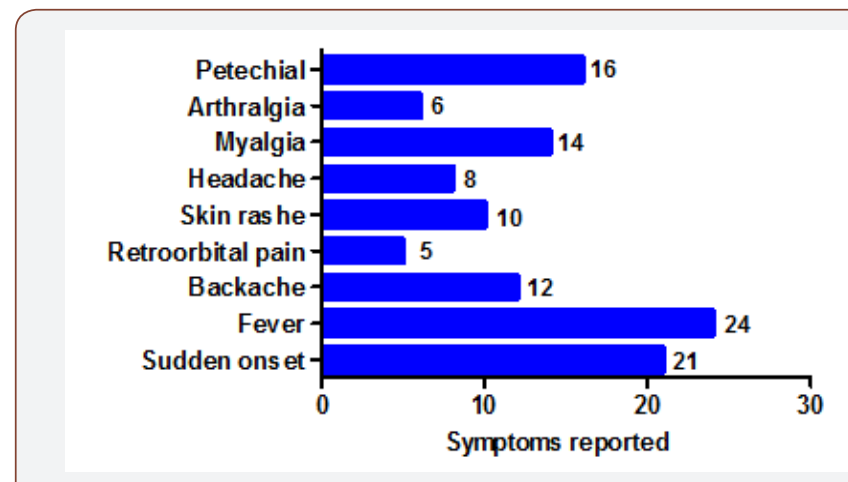

Figure 1: Clinical signs and symptoms of patients with arbovirus infection characteristics.

Our group, in collaboration with clinical care services, observed a great deal of confusion in the clinical diagnosis of arboviruses. This is due to numerous cases where patients presented characteristics of ZIKV infection, but it was not possible to confirm this infection by observation of signs and symptoms alone. In our studies of 25 patients with a clinical diagnosis of ZIKV infection were evaluated during the ZIKV and CHIKV outbreak in the period from January to August 2016. However, we did not obtain a positive result for the isolation of arboviruses in all analyzed samples, only concluding the isolation of CHIKV in 3 of the 25 collected samples. As can be observed in Figure 1, the same person may present symptoms characteristic of more than one arboviroses, demonstrating that even with the use of diagnosis may not be possible. This situation 
has occurred for both CHIKV and DENV infection, although symptoms caused by viruses such as Mayaro may be unknown by some health professionals as they are not common (Figure 1).

Cheap diagnostic methods with high reliability are long awaited and their availability would be very timely. Many groups have been working intensively to develop new strategies in the control and treatment of viral infection to reduce the risk of more severe clinical forms since there are no antivirals or vaccines available for clinical use [9]. We believe that more accurate diagnoses and better clinical knowledge of arboviruses can contribute to a significant reduction of more severe outbreaks or offer better control of sporadic outbreaks of these diseases.

\section{Acknowledgment}

None.

\section{Conflict of Interest}

No conflict of interest.

\section{References}

1. Gubler DJ (2002) The global emergence/resurgence of arboviral diseases as public health problems. Arch Med Res 33(4): 330-42.
2. Donalisio MR, Freitas ARR, Zuben APBV (2017) Arboviruses emerging in Brazil: challenges for clinic and implications for public health. Rev Saude Publica 51: 30 .

3. Basore K, Earnest JT, Diamond MS, Fremont DH (2018) Structural characterization of broadly neutralizing alphavirus antibodies. J Immunol 200(1): 126-133.

4. Alva Urcia C, Aguilar Luis MA, Palomares Reyes C, et al. (2017) Emerging and reemerging arboviruses: A new threat in Eastern Peru. PloS one 12(11): e0187897.

5. Colombo TE, Estofolete CF, Reis AFN, et al. (2017) Clinical, laboratory and virological data from suspected ZIKV patients in an endemic arbovirus area. J Clin Virol 96: 20-25.

6. Sánchez Duque JA, Rodríguez Morales AJ, Trujillo AM, Cardona Ospina JA, Villamil Gómez WE (2018) Cocirculation and Coinfection Associated to Zika Virus in the Americas.

7. Rückert C, Weger Lucarelli J, Garcia Luna SM, Young MC, Byas AD, et al. (2017) Impact of simultaneous exposure to arboviruses on infection and transmission by Aedes aegypti mosquitoes. Nat Commun 8: 15412.

8. Paixão ES, Teixeira MG, Rodrigues LC (2018) Zika, chikungunya and dengue: the causes and threats of new and re-emerging arboviral diseases. BMJ global health 3(Suppl 1): e000530.

9. Zmurko J, Marques RE, Schols D, Verbeken E, Kaptein SJ, et al. (2016) The Viral Polymerase Inhibitor 7-Deaza-2'-C-Methyladenosine Is a Potent Inhibitor of In Vitro Zika Virus Replication and Delays Disease Progression in a Robust Mouse Infection Model. PLOS Negl Trop Dis 10(5): e0004695. 